\title{
One-proton breakup reactions of proton-rich $p(s d)$ - shell nuclei for relevant reaction rates in explosive hydrogen burning
}

\author{
Adriana Banu for the E491 collaboration' \\ James Madison University, Department of Physics and Astronomy \\ 901 Carrier Dive, MSC 4502, Harrisonburg, VA 22807, USA \\ E-mail: banula@jmu.edu
}

To understand explosive hydrogen burning in stars and explore various explosive scenarios, such as classical novae or type I X-ray bursts (XRBs), reliable reaction rates are needed. The cross sections of radiative proton capture reactions on near drip line nuclei are necessary for the determination of the reaction rates, but cannot be measured directly. We discuss the use of oneproton removal reactions at intermediate energies as an indirect method in estimating (p, $\gamma$ ) reaction rates.

A cocktail beam of neutron-deficient nuclei was obtained by fragmentation of a 95 $\mathrm{MeV} /$ nucleon ${ }^{32} \mathrm{~S}$ primary beam provided by the Coupled Cyclotron Facility at GANIL. Longitudinal momentum distributions of the breakup fragments, in coincidence with $\gamma$ rays emitted by the fragments, were measured.

Results for one-proton nuclear breakup of ${ }^{24} \mathrm{Si},{ }^{23} \mathrm{Al},{ }^{22} \mathrm{Mg},{ }^{20,21} \mathrm{Na},{ }^{19,20} \mathrm{Ne},{ }^{18} \mathrm{~F},{ }^{16,17} \mathrm{O}$ will be reported in comparison with extended Glauber calculations. Determination of the corresponding Asymptotic Normalization Coefficients (ANCs) and their use to infer stellar reaction rates with astrophysical implications for explosive hydrogen burning in novae or type I XRBs are presented.

XII International Symposium on Nuclei in the Cosmos August 5-12, 2012

Cairns, Australia

Speaker for E491 collaboration: A. Banu, L. Trache, R. E. Tribble (Cyclotron Institute, TAMU), F. Carstoiu, F. Negoita, F. Rotaru (IFIN-HH), N. A. Orr, N. L. Achouri, B. Laurent, B. T. Roeder (LPC-ENSICAEN), P. Roussel-Chomaz, L. Gaudefroy (GANIL), M. Chartier, M. Dimmock, B. Fernandez-Dominguez, S. Paschalis, E. S.Paul, M. Petri, B. Pietras (Univ. of Liverpool), W. N. Catford, . Patterson, J. S. Thomas (Univ. of Surrey), R. C. Lemmon (Daresbury Laboratory), M. Freer (Univ. of Birmingham), M. Labiche (Univ. of the West Scotland), M. Horoi (Central Michigan Univ.), and A. Bonaccorso (INFN-Pisa, Univ. of Pisa) 


\section{Introduction}

To make reliable predictions for the nucleosynthesis of chemical elements and the evolution of stars and galaxies, we need several types of data. This includes the reaction rates of the nuclear reactions relevant in nucleosynthesis, especially explosive processes such as classical novae and type I X-ray bursts (XRBs). Stellar nucleosynthesis processes typically may involve unstable nuclei, and we have currently only limited knowledge about their corresponding reaction rates. Direct measurements for nuclear astrophysics involving unstable nuclei are difficult. Hence, only very few (e.g. [1]) have been done on unstable nuclei to date. This problem has led to the development and use of indirect methods in nuclear astrophysics such as Coulomb dissociation (see, e.g. T. Motobayashi et al. Phys. Rev. Lett. 73 (2680), 1994), transfer reaction (the ANC method) (see, e.g. A.M. Mukhamedzanov, C. A. Gagliardi, R. E. Tribble, Phys. Rev. C 63 (024612) 2001), the Trojan horse method (see, e.g. C. Spitaleri et al, Phys. Rev. C 60 (055802) 1998).

Work done recently and in the last decade at several laboratories has demonstrated that nuclear breakup reactions can be a good and reliable spectroscopic tool [2-7] and can be used to obtain information of importance to nuclear astrophysics [8-11]. In particular, we can use data from the nuclear one-proton breakup of nuclei like $\mathrm{X} \rightarrow \mathrm{Y}+p$ to extract asymptotic normalization coefficients (ANCs) for the $\mathrm{Y}+p$ system. This information is sufficient to determine the non-resonant contribution to the reaction rate for radiative proton capture $Y(p, \gamma) X$ at stellar temperatures, which is a major contribution at stellar energies in a large number of reactions with relevance in nuclear astrophysics, in particular for explosive H-burning. The method involves breakup in the nuclear field of the target (as opposed to the Coulomb field) and it is peripheral for a large range of projectile energies. These energies depend on the particular nuclei (mostly on the binding energy and the orbital momentum of the involved single-particle orbital), but are generally in the intermedate energy range. The use of nuclear breakup has the experiemental advantage that it can use cocktail beams of moderate or low intensities. The breakup cross sections are much larger (about $100 \mathrm{mb}$ ) than those for proton transfer reactions (a few mb), the alternative method used to determine ANCs which also requires beams of high purity and intensity.

The work discussed here refers to a recent one-proton breakup experiment performed at GANIL with a radiaoctive cocktail beam centered around ${ }^{23} \mathrm{Al}$ at $50 \mathrm{MeV} /$ nucleon. The original motivation of this work was to study the ${ }^{22} \mathrm{Mg}(\mathrm{p}, \gamma)^{23} \mathrm{Al}$ reaction for its astrophysical implications related to the ${ }^{22} \mathrm{Na}$ nucleosynthesis in $\mathrm{O}-\mathrm{Ne}$ novae. Moreover, the ${ }^{23} \mathrm{Al}(\mathrm{p}, \gamma)^{24} \mathrm{Si}$ reaction was also investigated in the same experiment since its rate can affect both the ${ }^{22} \mathrm{Na}$ abudance and the XRB total energy output as well as the nucleosynthesis occuring in the type I XRBs.

In addition to the data relevant for nuclear astrophysics, we expect that the results of this work contribute significantly to the understanding of the reaction mechanism at intermediate energies and to the develpoment of theoretical reaction models and codes. 


\section{Experiment}

As aforementioned, the experiment discussed here was carried out recently at the GANIL coupled cyclotron facility. A cocktail of secondary beams was produced via the fragmentation of a ${ }^{32} \mathrm{~S}$ primary beam at $95 \mathrm{MeV} /$ nucleon impinging on a carbon target. Several ion species such as ${ }^{16,17} \mathrm{O},{ }^{18} \mathrm{~F},{ }^{19,20} \mathrm{Ne},{ }^{20,21} \mathrm{Na},{ }^{22} \mathrm{Mg},{ }^{23} \mathrm{Al},{ }^{24} \mathrm{Si}$ were obtained with energies between 35 and $60 \mathrm{MeV} /$ nucleon and intensities ranging from 30 to 7000 pps. A secondary reaction target also of carbon was used. To measure the longitudinal momentum distributions of the breakup residues, the SPEG energy-loss spectrometer was employed. SPEG's overall momentum acceptance ws $7 \%$, which permitted to measure in one setting the momentum distributions of the fragments resulting from one-proton breakup of all nuclei of interest in the secondary cocktail beam. Furthermore, the large angular acceptance of the spectrometer $\left(4^{\circ}\right.$ in both horizontal and vertical planes) provided for complete collection of the breakup fragments, obviating any ambiguities in the integrated cross sections and longitudinal momentum distributions. More experimental details can be found in Refs. $[9,10]$. In Figure 1, we plot the ion identification of the secondary cocktail beams.

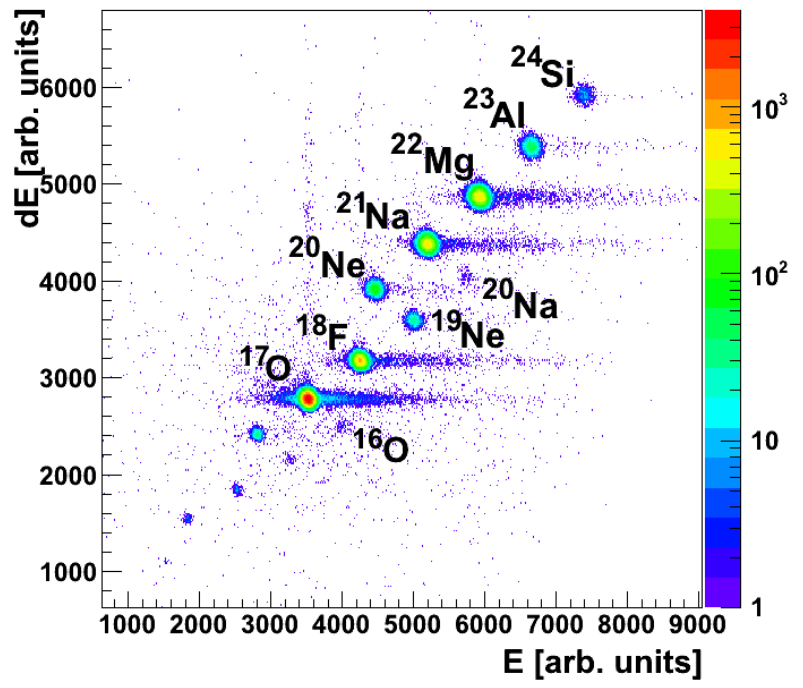

Figure 1: Particle identification of the seondary cocktail beam plotted as energy loss vs. residual energy detected with SPEG's ionization chamber and thick plastic scintillator, respectively. See Refs. [9,10] for details.

\section{Results}

We present here published results for the one-proton breakup of ${ }^{24} \mathrm{Si}$ and ${ }^{23} \mathrm{Al}$ and new preliminary results for 8 more one-proton breakup cases studied in our work: ${ }^{22} \mathrm{Mg},{ }^{20,21} \mathrm{Na}$, ${ }^{19,20} \mathrm{Ne},{ }^{18} \mathrm{~F}$ and ${ }^{16,17} \mathrm{O}$. We have determined the ground state composition of nuclear wave functions for the secondary ion beam species. This was done measuring longitudinal momentum distribution of the corresponding breakup residues, left excited after one-proton breakup reactions occured, in coincidence with the $\gamma$ rays de-exciting them.

To describe the nuclear component of the one-proton breakup (the dominant mechanism for a carbon target), an extended version of the Glauber model, which incorporates second-order noneikonal corrections in the evaluation of the scattering amplitude that defines the stripping 
and diffraction transition operators, has been employed. The formalism used is described in Ref. [3].

The low-lying nuclear structure of the nuclei in the secondary cocktail beams that undergo one-proton breakup in the nuclear field of the carbon target can be assumed to be that of a core (the breakup fragment) plus a valence proton. In the particular case of ${ }^{24} \mathrm{Si}$, because there are no known particle-bound excited states in ${ }^{23} \mathrm{Al}$, the ${ }^{24} \mathrm{Si} \mathrm{J}^{\pi}=0^{+}$ground-state wave function will only have a single configuration that couples a proton to the core ground state, ${ }^{23} \mathrm{Al}\left(5 / 2^{+}\right)_{\mathrm{gs}} \otimes \pi$ $1 \mathrm{~d}_{5 / 2}$, which is the configuration of astrophysical interest for the nonresonant (direct) radiative capture ${ }^{23} \mathrm{Al}(\mathrm{p}, \gamma)^{24} \mathrm{Si}$. Hence, no $\gamma$ rays would be expected in coincidence with the measured ${ }^{23} \mathrm{Al}$ residues, and indeed, none were detected. Unlike the case of ${ }^{24} \mathrm{Si}$, in the case of ${ }^{23} \mathrm{Al}$ we have identified three $\gamma$-ray lines corresponding to the transitions $2^{+}{ }_{1} \rightarrow 0^{+}{ }_{\mathrm{gs}}, 4^{+}{ }_{1} \rightarrow 2^{+}{ }_{1}, 4^{+}{ }_{2} \rightarrow 4^{+}{ }_{1}$ in the de-excitation of the ${ }^{22} \mathrm{Mg}$ breakup core (see Ref. [9]). This leads to a configuration mixing in the ground state of ${ }^{23} \mathrm{Al}$ of the type: $\left.\left.\left.\left.\right|^{23} \mathrm{Al}_{\mathrm{gs}}\left(5 / 2^{+}\right)\right\rangle=\left.A_{0}\right|^{22} \mathrm{Mg}\left(0^{+} \mathrm{gs}^{\mathrm{s}}\right) \otimes \pi 1 \mathrm{~d}_{5 / 2}\right\rangle+\left.A_{l}\right|^{22} \mathrm{Mg}\left(2^{+}{ }_{1}\right) \otimes \pi 1 \mathrm{~d}_{5 / 2}\right\rangle+$ $\left.\left.\left.A_{2}\right|^{22} \operatorname{Mg}\left(4^{+}{ }_{1}\right) \otimes \pi 1 \mathrm{~d}_{5 / 2}\right\rangle+\left.A_{3}\right|^{22} \operatorname{Mg}\left(4^{+}{ }_{2}\right) \otimes \pi 1 \mathrm{~d}_{5 / 2}\right\rangle$, where $A_{i}$ (i $=0$-3) represent the spectroscopic amplitudes of each of the four configurations. The corresponding exclusive longitudinal momentum distributions measured in coincidence with these $\gamma$ rays are shown in Fig. 3 of Ref. [9]. The momentum distribution corresponding to ${ }^{22} \mathrm{Mg}$ ground state, which defines the configuration of astrophysical interest for the nonresonant radiative capture ${ }^{22} \mathrm{Mg}(\mathrm{p}, \gamma)^{23} \mathrm{Al}$, was derived by subtraction of the measured exclusive momnetum distributions from the measured inclusive distribution.

In Figure 2 are plotted the measured inclusive momentum distributions for both one-proton breakup cases of ${ }^{24} \mathrm{Si}$ and ${ }^{23} \mathrm{Al}$ along with theoretical distributions calculated in the extended Glauber model.
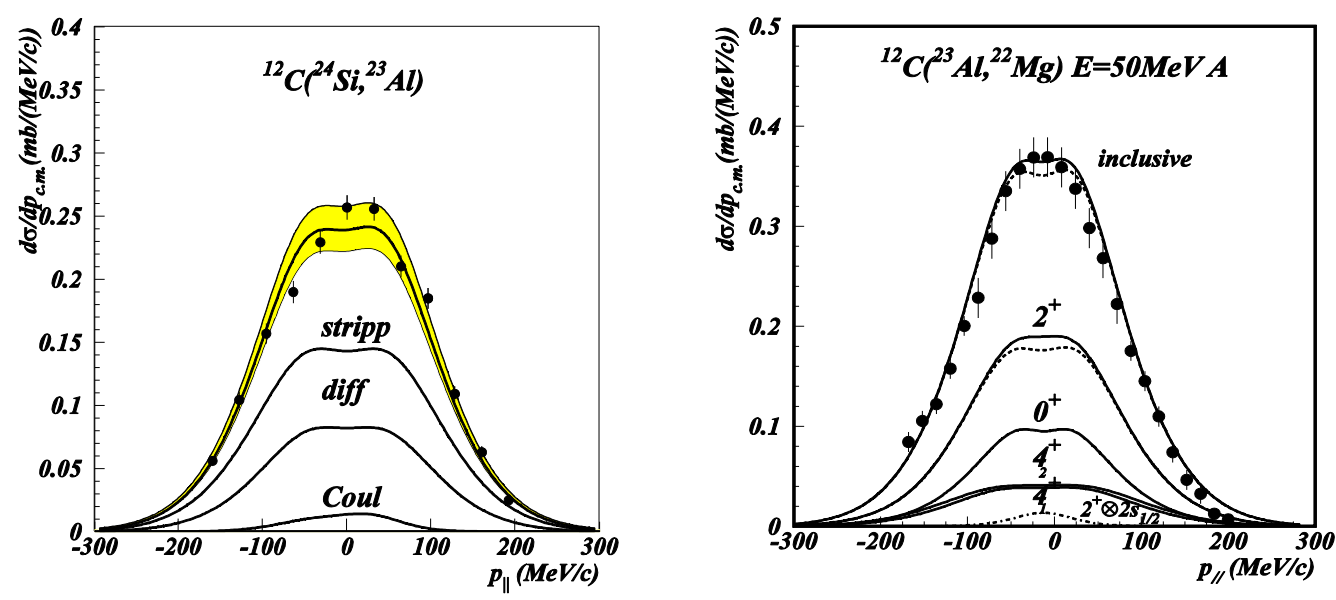

Figure 2: Experimental inclusive momentum distributions from one-proton breakup of ${ }^{24} \mathrm{Si}$ (left) and ${ }^{23} \mathrm{Al}$ (right) in the center of reference of the core nuclei ${ }^{23} \mathrm{Al}$ and ${ }^{22} \mathrm{Mg}$, respectively. In compariosn, are also shown the corresponding theoretical distribution calculated within the framework of the extended Glauber model. See Refs. $[9,10]$ for details. 
Preliminary results are shown in Figure 3 for eight new cases of one-proton breakup identified from the measured data of this work. Hence, in Figure 3 we present non-normalized longitudinal momentum distributions transformed in the reference center for the ${ }^{21} \mathrm{Na},{ }^{19,20} \mathrm{Ne}$, ${ }^{18,19} \mathrm{~F},{ }^{17} \mathrm{O},{ }^{15,16} \mathrm{~N}$ breakup cores. We also show the corresponding Doppler-corrected $\gamma$-ray spectra taken in coincidence with the measurement of the momentum distributions of the breakup fragments. This information is needed in order to disentangle the configuartion mixing in the ground state wave functions of the breakup fragments. This will lead to the experimental determination of the values for the corresponding spectroscopic factors, to be compared with values obtained from state-of-the-art large scale shell model calculations, as it was done for the two published cases of one-proton breakup of ${ }^{24} \mathrm{Si}$ and ${ }^{23} \mathrm{Al}$ of this work.
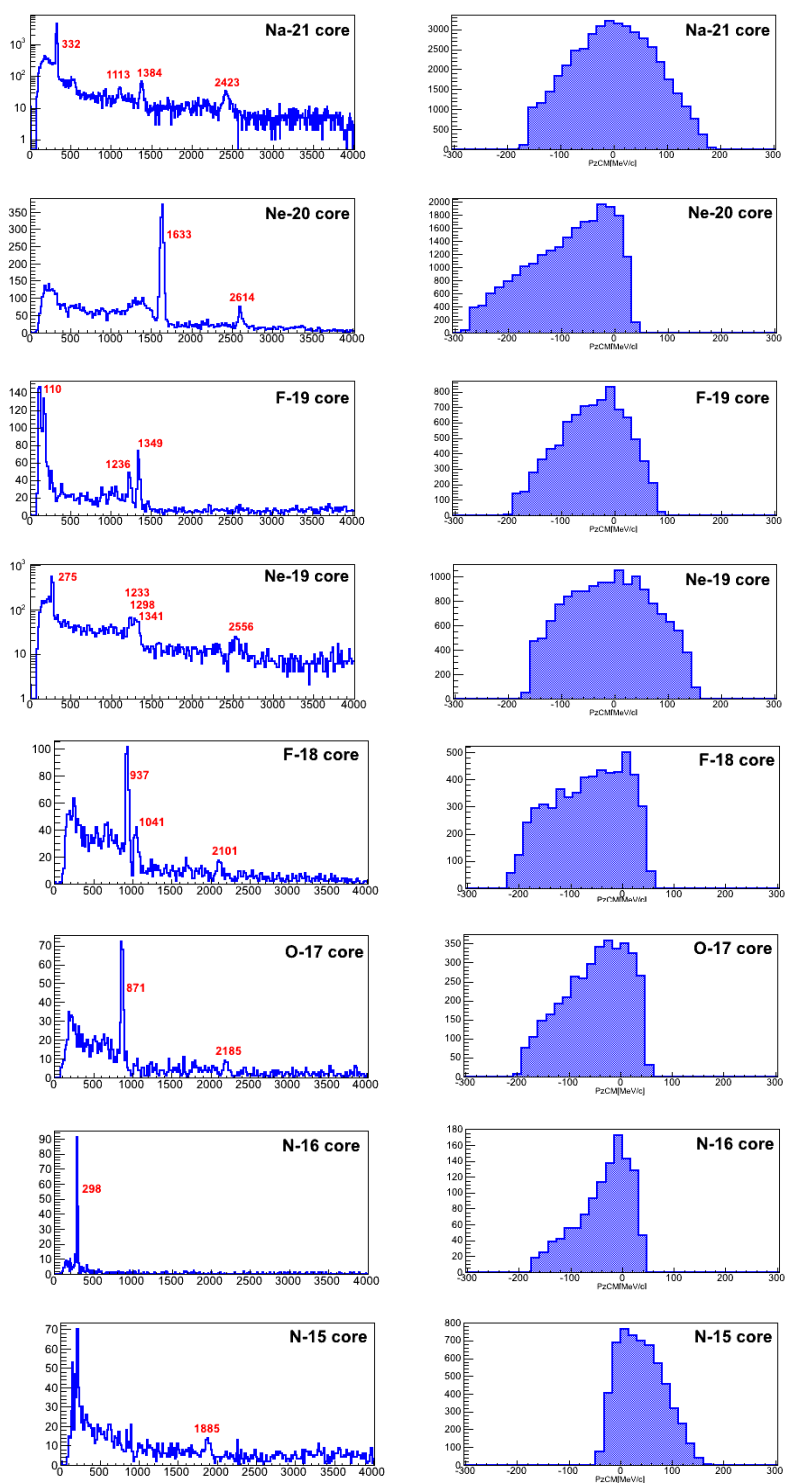

Figure 3: Experimental data on the longitudinal momentum distributions and Doppler-corrected $\gamma$-ray spectra for the core fragments corresponding to one-proton breakup of ${ }^{22} \mathrm{Mg},{ }^{20,21} \mathrm{Na},{ }^{19,20} \mathrm{Ne},{ }^{18} \mathrm{~F},{ }^{16,17} \mathrm{O}$. 
From the experimental spectroscopic factors, the asymptotic normalization coefficients can be evaluated, and thus the nonresonant (direct) component of the astrophysical $S$-factors characterizing the radiative proton captures of interest. See the published results for the reactions ${ }^{22} \mathrm{Mg}(\mathrm{p}, \gamma){ }^{23} \mathrm{Al}$ (Ref. [9]) and ${ }^{23} \mathrm{Al}(\mathrm{p}, \gamma)^{24} \mathrm{Si}$ (Ref. [10]). Work is in progress to evaluate the $(\mathrm{p}, \gamma)$ reaction rates corresponding to the remaining eight one-proton breakup cases identified in the data of this study.

\section{References}

[1] A. Sallaska, A. Garcia, D. W. Storm, T. A. D. Brown, C. Ruiz, K. A. Snover, D. F. Ottelwell, L. Buchmann, C. Vockenhube et al., Direct measurements of ${ }^{22} \mathrm{Na}(\mathrm{p}, \gamma)^{23} \mathrm{Mg}$ resonances and consequences for ${ }^{22}$ Na production in classical novae, Phys. Rev. Lett. 105 (152501) 2010

[2] P. G. Hansen and B. M. Sherrill, Reactions and single-particle structure of nuclei near the drip lines, Nucl. Phys. A693 (133) 2001

[3] E. Sauvan, F. Carstoiu, N. A. Orr, J. S. Winfield, M. Freer, J. C. Angelique, W. N. Catford, N. M. Clarke, N. Curtis, S. Grevy et al., One-neutron removal reactions on light neutron-rich nuclei, Phys. Rev. C 69 (044603) 2004

[4] A. Gade, P. Adrich, D. Bazin, M. D. Bowen, B. A. Brown, C. M. Campbell, J. M. Cook, T. Glasmacher, P. G. Hansen, K. Hosier et al., Reduction of spectroscopic strength: Weakly-bound and strongly-bound single-particle states studied using one-nucleon knockout reactions, Phys. Rev. C 77 (044306) 2008

[5] R. Shane, R. J. Charity, L. G. Sobotka, D. Bazin, B. A. Brown, A. Gade, G. F. Grinyer, S. McDaniel, A. Ratkiewicz, D. Weisshaar et al., Proton and Neutron knockout from ${ }^{36}$ Ca, Phys. Rev. C 85 (064612) 2012

[6] F. Flavigny, A. Obertelli, A. Bonaccorso, G. F. Grinyer, C. Louchart, L. Nalpas, and A. Signoracci, Nonsudden limits of heavy-ion induced knockout reactions, Phys. Rev. Lett. 108 (252501) 2012

[7] G. F. Grinyer, D. Bazin, A. Gade, J. A. Tostevin, P. Adrich, M. D. Bowen, B. A. Brow, C. M. Campbell, J. M. Cook, T. Glasmacher et al., Systematic study of p-shell nuclei via single-nucleon knockout reactions, Phys. Rev. C 86 (024315) 2012

[8] L. Trache, F. Carstoiu, C. A. Gagliardi, and R. E. Tribble, Asymptotic normalization coefficient of ${ }^{8} B$ from breakup reactions and the $S_{17}$ astrophysical factor, Phys. Rev. Lett. 87 (271102) 2001

[9] A. Banu, L. Trache, F. Carstoiu, N. L. Achouri, A. Bonaccorso, W. N. Catford, M. Chartier, M. Dimmock, B. Fernandez-Dominguez, M. Freer et al., Structure of ${ }^{23}$ Al from the one-proton breakup reaction and astrophysical implications, Phys. Rev. C 84 (015803) 2011

[10] A. Banu, F. Carstoiu, N. L. Achouri, W. N. Catford, M. Chartier, B. Fernandez-Dominguez, M. Horoi, B. Laurent, N. A. Orr, S. Pachalis et al., One-proton breakup of ${ }^{24} \mathrm{Si}$ and the ${ }^{23} \mathrm{Al}(\mathrm{p}, \gamma)^{24} \mathrm{Si}$ reaction in type I X-ray bursts, Phys. Rev. C 86 (015806) 2012

[11] T. Fukui, K. Ogata, K. Minomo, M. Yahiro, Determination of the ${ }^{8} B(p, \gamma)^{9} C$ reaction rate from ${ }^{9}$ C breakup, Phys. Rev. C 86 (022801)(R) 2012 